\title{
Non-typhoidal Salmonella DNA traces in gallbladder cancer
}

\author{
Prajish lyer ${ }^{1}$, Savio George Barreto², Bikram Sahoo ${ }^{1}$, Pratik Chandrani ${ }^{1}$, Mukta R. Ramadwar ${ }^{3}$, \\ Shailesh V. Shrikhande ${ }^{4}$ and Amit Dutt ${ }^{*}$ (D)
}

\begin{abstract}
Background: We earlier proposed a genetic model for gallbladder carcinogenesis and its dissemination cascade. However, the association of gallbladder cancer and 'inflammatory stimulus' to drive the initial cascade in the model remained unclear. A recent study suggested infection with Salmonella can lead to changes in the host signalling pathways in gallbladder cancer.

Findings: We examined the whole exomes of 26 primary gall bladder tumour and paired normal samples for presence of 143 HPV (Human papilloma virus) types along with 6 common Salmonella serotypes (S. typhi Ty2, S. typhi CT18, S. typhimurium LT2, S. choleraesuis SCB67, S. paratyphi TCC, and S. paratyphi SPB7) using a computational subtraction pipeline based on the HPVDetector, we recently described. Based on our evaluation of 26 whole exome gallbladder primary tumours and matched normal samples: association of typhoidal Salmonella species were found in 11 of 26 gallbladder cancer samples, and non-typhoidal Salmonella species in 12 of 26 gallbladder cancer, with 6 samples were found co-infected with both.

Conclusions: We present the first evidence to support the association of non-typhoidal Salmonella species along with typhoidal strains in gallbladder cancer. Salmonella infection in the chronic carrier state fits the role of the 'inflammatory stimulus' in the genetic model for gallbladder carcinogenesis that may play a role in gallbladder cancer analogous to Helicobacter pylori in gastric cancer.
\end{abstract}

Keywords: Salmonella, Gall bladder cancer, Non-typhoidal, Inflammation

\section{Background}

Gallbladder cancer with its attendant poor prognosis [1], despite the performance of good surgery when feasible [2], lends itself to the need for further research. Scanu et al. recently provided a novel mechanism by which chronic Salmonella infection of the gallbladder can lead to changes in the host signalling pathways triggering cellular transformations resembling the evolution of gallbladder cancer [3]. This is consistent with earlier epidemiological findings that support the association of typhoidal Salmonella typhi and $S$. paratyphi with the risk of gallbladder cancer [4-7]. However, non typhoidal species (S. typhimurium, $S$ choleraesuis) that elicits a stronger host immune response

\footnotetext{
* Correspondence: adutt@actrec.gov.in

${ }^{1}$ Advanced Centre for Treatment, Research and Education in Cancer,

Kharghar, Navi Mumbai, India

Full list of author information is available at the end of the article
}

compared to the typhoidal species and are linked to a systemic illness have as yet not reported to be associated with gallbladder disease or cancer [8]. Interestingly, in the Indian subcontinent where gallbladder incidence rates are amongst the highest in the world, Salmonella infection is also considerably severe [9].

We have previously proposed a model for gallbladder carcinogenesis based on the current understanding of the tumour biology and associated inflammation [10]. However, there remain lacunae in the model that need to be clarified. For instance, what is the inflammatory stimulus' that drives the initial cascade of an upregulation of inflammatory markers that is characterised by an increase in protective mucins as well as a strange divergence of inflammatory markers thereafter from the stage of in situ to invasive cancer? Here in this study we examined the exomes of primary gall bladder tumour and paired normal samples for presence of 6 common Salmonella serotypes with available genome information $(S$. 
typhi Ty2, S. typhi CT18, S. typhimurium LT2, S. choleraesuis SCB67, S. paratyphi TCC, and S. paratyphi SPB7) using a computational subtraction pipeline based on the HPVDetector tool [11]

\section{Methods}

\section{Patient sample collection}

Twenty six fresh frozen primary tumour and matched normal tissues were obtained from the tissue repository of Tata Memorial hospital (TMH). The Institutional Review Board (IRB) and the Ethics Committee (EC) of Tata Memorial Centre (TMC) - Advanced Centre for Treatment, Research and Education in Cancer (ACTREC) (Mumbai, India) approved the project (\#104). Since this was a retrospective analysis, the IRB and the EC waived the need for an informed consent. Patients were randomly selected based on the availability of fresh frozen tissues. The patient characteristics including age, gender, gall stone status and histopathology were recorded.

\section{PCR analysis for Salmonella isolates}

The PCR method used for Salmonella detection has been previously described [12]. Nested PCR was carried out in a $25 \mu \mathrm{l}$ volume containing $10 \mu \mathrm{l}$ KAPA $2 \mathrm{X}$ ready mix master-mix (KapaBiosystems catalog no-KK1024), 10 pmol primer and $100 \mathrm{ng}$ of genomic DNA. Following the first round of PCR $\left(94{ }^{\circ} \mathrm{C}\right.$ for $1 \mathrm{~min}, 55^{\circ} \mathrm{C}$ for $1 \mathrm{~min} 15 \mathrm{~s}$, $72{ }^{\circ} \mathrm{C}$ for $3 \mathrm{~min}-40$ cycles) with ST1 and ST2 primers, $5 \mu \mathrm{l} \mathrm{PCR}$ product was used as template for nested PCR using ST3 and ST4 primers $\left(94{ }^{\circ} \mathrm{C}\right.$ for $1 \mathrm{~min}, 68{ }^{\circ} \mathrm{C}$ for $1 \mathrm{~min} 15 \mathrm{~s}, 72{ }^{\circ} \mathrm{C}$ for $3 \mathrm{~min}-40$ cycles). We also performed validation of Salmonella sequences using read specific primers. The PCR conditions $-94{ }^{\circ} \mathrm{C}$ for $1 \mathrm{~min}, 59^{\circ} \mathrm{C}$ for $30 \mathrm{~s}, 72{ }^{\circ} \mathrm{C}$ for $45 \mathrm{~s}-$ for 30 cycles. The primer sequences are detailed in Additional file 1: Table S2.

\section{Sequencing and analysis}

Exome capture and library preparation was performed using Agilent Sure select in-solution (low-input capture$500 \mathrm{ng}$ ) target enrichment technology. Genomic DNA was sheared and size selected (150-200 bp) and ligated to adaptors and run on Illumina Nextseq 500 platform to generate $150 \mathrm{bp}$ paired end reads at a coverage of $100 \mathrm{X}$ and above. To detect Salmonella traces, the HPVDetector pipeline was used, as described previously [11]. Briefly, reads were aligned against six known Salmonella species genomes in addition of the HPVDetector nce data set of $143 \mathrm{HPV}$ types, as downloaded from the National Centre for Biotechnology Information (NCBI), using BWA (Burrows wheeler algorithm) aligner (v0.6.2). All reference sequences were annotated and concatenated to compose a multi-fasta sequences using bio-perl modules. The alignment files were parsed using UNIX shell program to detect the types of Salmonella represented by at least one read that aligned to a particular Salmonella type with high confidence.

\section{Results \\ HPVDetector pipeline identifies Salmonella sequence present in gallbladder cancer samples}

We performed PCR based analysis of 26 gallbladder tumour and paired normal samples to detect the presence of Salmonella DNA using pan primers, as described earlier [12]. None of the gallbladder samples were found to be positive for Salmonella (data not shown). As a next step, whole exome data for these 26 samples (generated in house, manuscript in make) were analysed to detect Salmonella traces using HPVDetector pipeline, modified to include additional genome sequence of 6 common Salmonella isolates. The computational approach, in brief, subtracts all reads that align to human genome and aligns remaining reads to HPV and Salmonella reference database from NCBI. While HPV16 was detected in 1 gallbladder sample, Salmonella isolates were found across multiple samples: S. typhi Ty2 (3 samples), S. typhi CT18 (6 samples), S. typhimurium LT2 (10 samples), S. choleraesuis SCB67 (5 samples), S. paratyphi TCC (3 samples), and S. paratyphi SPB7 (7 samples). In total, Salmonella reads were found in 19 of 26 gallbladder tissues (tumor as well as adjacent normal tissues). Interestingly, 10 of 19 samples were co-infected with multiple isolates independent of gallstone status or gender of the patients, as shown in Fig. 1.

\section{Annotation of the Salmonella reads found in gallbladder cancer samples}

Variable number of overlapping reads of variant lengths for each isolate were assembled into contigs based on Clustal X2 multiple alignment as shown in Additional file 2: Figure S1. The unique stretch of contigs generated were annotated based on gene annotation database of Salmonella isolates from NCBI (National centre for biotechnology information) database. 114 reads of multiple Salmonella isolates were found in 19 of 26 samples analyzed. 47 of 114 reads of Salmonella ORF (open reading frame) were identified as encoding for bacterium genes known to be involved in metabolism and those related to the toxin-antitoxin system. Rest of the reads aligned to the Salmonella ribosomal genes, understandably due to their relatively higher abundance (Additional file 3: Table S1).

\section{HPVDetector pipeline is specific and highly sensitive to detect true Salmonella traces}

To assess specificity of our assay, we re-analyzed whole exome data of all samples by taking their reverse (not complement) to simulate random sequence, but retaining composition of nucleotides and genome complexity, using an in-house perl script, as described earlier [11]. 


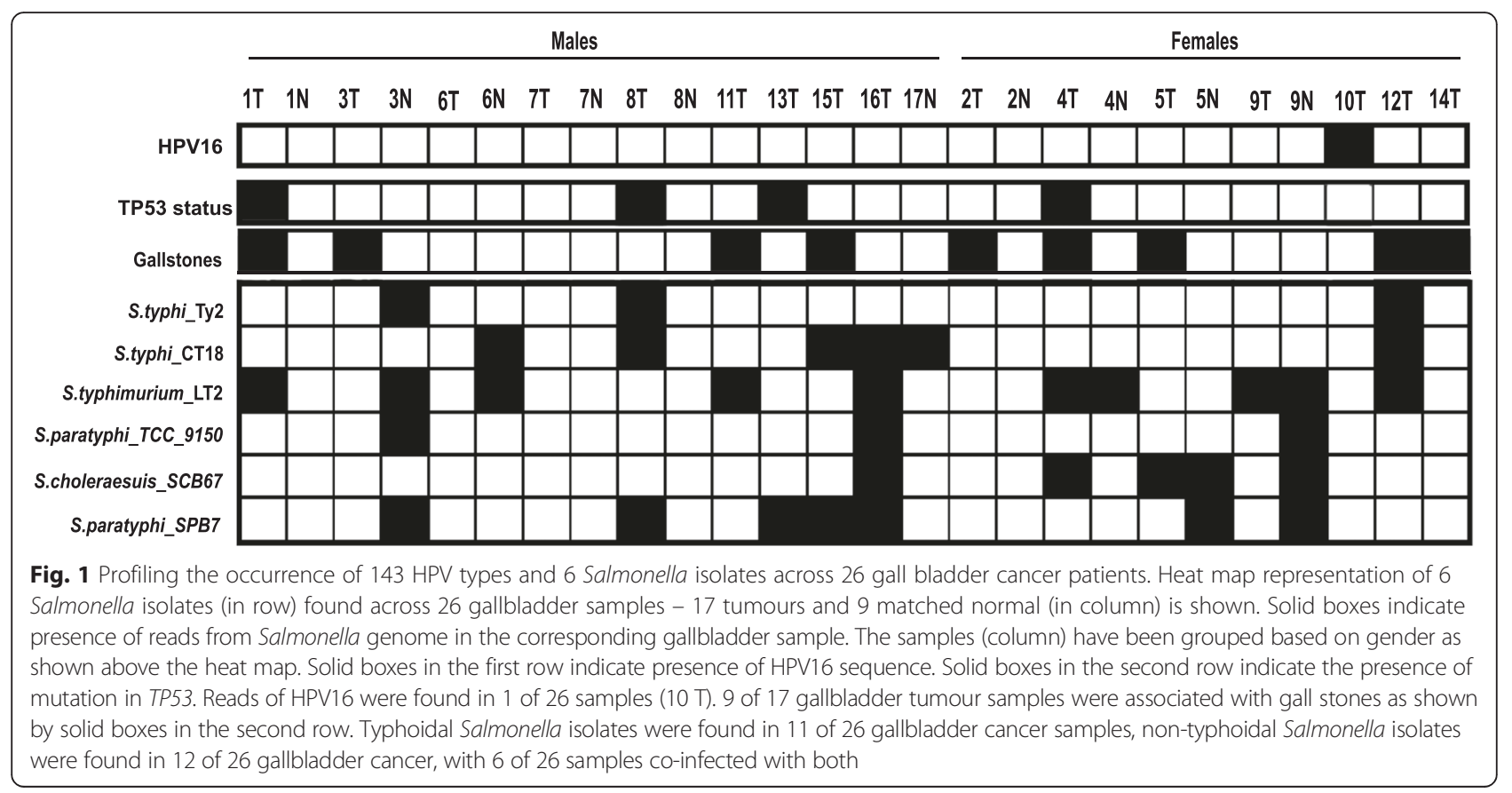

We found no spurious Salmonella reads when the primary tumour whole exome sequence was reversed in any of the 26 samples, suggesting the computational pipeline used was specific to detect Salmonella traces. (Additional file 4: Figure S2A). To test the sensitivity of our assay, raw FASTQ file of a primary tumour sample $16 \mathrm{~T}$ that was found positive for Salmonella reads was downsampled to $1 \mathrm{X}, 5 \mathrm{X}, 10 \mathrm{X}, 15 \mathrm{X}, 25 \mathrm{X}, 50 \mathrm{X}, 75 \mathrm{X}$ and $100 \mathrm{X}$ coverage using Picard Toolkit's DownsampleSam function (http://broadinstitute.github.io/picard/), as described earlier [11]. The resulting FASTQ files were analysed for detection of Salmonella reads using the HPVDetector pipeline. Distinct Salmonella reads were detected at as low as 10X whole exome coverage that increased linearly (Additional file 4: Figure S2B).

\section{Sanger validation of Salmonella reads identified in gallbladder cancer samples}

We have attempted to validate the presence of Salmonella read sequences identified by HPVDetector in 4 of 16 Salmonella positive samples using Sanger sequencing (Additional file 5: Figure S3).

\section{Discussion}

We examined Salmonella and HPV DNA sequences in gallbladder tumours and paired normals, The high incidence of Salmonella sequence found in 16 of 26 samples analysed in the study suggests a possible role of Salmonella infection in gallbladder cancer analogous to Helicobacter pylori in gastric cancer and Fusobacterium in colon cancer $[13,14]$. We demonstrate the presence of typhoidal Salmonella species in 11 of 26 gallbladder cancer samples, consistent with as known earlier. In addition, we present the first evidence to support the association of even nontyphoidal Salmonella species in 12 of 26 gallbladder cancer, with 6 of 26 samples co-infected with typhoidal as well as non typhoidal Salmonella isolates.

Systemic inflammation is known to be associated with a poor prognosis in gallbladder cancer [15]. Owing to the ability of Salmonella infection to stimulate a host response, it is likely that these bacteria are able to provide the continued 'inflammatory stimulus' necessary for carcinogenesis. Recent reports suggest that Salmonella infections promote malignant transformation in genetically predisposed mice, murine gall bladder organoids and fibroblasts with TP53 mutations [3]. We observed 4 of 16 Salmonella positive samples harboured TP53 mutations while we did not observe TP53 mutations in Salmonella negative samples. Salmonella isolates in the chronic carrier state thus fits the role of the 'inflammatory stimulus' in the genetic model for gallbladder carcinogenesis and its dissemination cascade, which may trigger transformation through chronic inflammation, but not for maintenance of tumourigenesis [10].

The focus of treatment in typhoid-endemic countries such as India has historically been solely on eliminating typhoidal Salmonella species often underestimating the contribution of the non-typhoidal isolates that show an inherent higher resistance to the standard antibiotics [8] resulting in their ability to lead to chronic carrier state in humans. The presence of non-typhoidal Salmonella species in our study highlights that in typhoid-, as well as gallbladder cancer-endemic countries such as India and other similar countries, efforts must be directed not only 
at treating typhoid fever, but also diagnosing and appropriately managing non-typhoidal Salmonella species. This simple approach could reduce the chronic carrier state of these species in humans, which by our hypothesis may be contributing to the inflammatory stimulus driving gallbladder carcinogenesis. Thus, this simple strategy may help reduce in the incidence of gallbladder cancer. While this study validates and extends the association of Salmonella with gall bladder carcinoma, further study is required to establish the causality of infection to the disease.

\section{Additional files}

Additional file 1: Table S2. Primer sequences used for detection of Salmonella (PDF $122 \mathrm{~kb}$ )

Additional file 2: Figure S1. Abundance and annotation of Salmonella reads found across the 16 of 26 gall bladder cancer samples. Heat map representation of individual Salmonella reads (in rows) identified from 6 different isolates found across the 16 gall bladder cancer samples (in column) is shown. Variable length and number of overlapping reads, each of $150 \mathrm{bp}$ obtained from paired end Illumina sequence for each isolate, were assembled into contigs based on Clustal X2 multiple alignment. The unique total length of contigs generated is shown in second column reflecting the total length of the gene covered in the study. The contigs generated were annotated based on gene annotation database of Salmonella isolates from NCBI database. A representative general class for all genes identified is shown in the third column. (PDF $11190 \mathrm{~kb}$ )

Additional file 3: Table S1. Detailed annotation table of read sequences of different Salmonella species identified across gallbladder cancer patient samples. (PDF 348 kb)

Additional file 4: Figure S2. Specificity and Sensitivity for detection of Salmonella reads in whole exome sequence of gallbladder samples. (A) Specificity for detection of Salmonella reads in whole exome sequence of gallbladder samples. Exome sequenced reads were reversed (not complement) to maintain the genome complexity and used an input file to detect random Salmonella reads. No Salmonella reads were found in the samples with reversed whole exome sequence. (B) Sensitivity for detection of Salmonella reads in gallbladder samples as a function of increasing genome sequence coverage. Gallbladder tumour sample $16 \mathrm{~T}$ with highest number of Salmonella reads was down-sampled to $1 x, 5 x, 10 x, 15 x, 25 x, 50 x$, $75 x$ and 100x. Salmonella reads were counted (black line) and plotted against increasing coverage of the genome on $x$-axis. (PDF $5395 \mathrm{~kb}$ )

Additional file 5: Figure S3. Sanger validation of Salmonella read sequences in gall bladder cancer samples Individual read sequences were PCR amplified and Sanger sequencing trace of individual read sequence with their blast output is represented in the figure. (PDF $1437 \mathrm{~kb}$ )

\section{Abbreviations}

HPV: human papilloma virus; NCBI: national centre for biotechnology information; ORF: open reading frame; PCR: polymerase chain reaction; BWA: Burrows wheeler aligner.

\section{Competing interests}

The authors declare that they have no competing interests

\section{Author's contributions}

P.I. and S.G.B contributed equally to this work; P.I., S.G.B, S.V.S. and A.D. designed research; P.I., B.S, P.C and M.R.R performed research; S.S. and M.R.R contributed primary tumor samples and their pathological review; P.I., S.G.B, B.S. and A.D. analyzed data; and P.I., S.G.B, and A.D. wrote the paper. All authors read and approved the final manuscript.

\section{Acknowledgements}

All members of the Dutt laboratory for critically reviewing the manuscript. A.D. is supported by an Intermediate Fellowship from the Wellcome Trust/ DBT India Alliance (IA/I/11/2500278), by a grant from DBT (BT/PR2372/AGR/ 36/696/2011), and intramural grants (IRB project 92 and 55). P.I and P.C are supported by senior research fellowship from ACTREC. The funders had no role in study design, data collection and analysis, decision to publish, or preparation of the manuscript.

\section{Author details}

${ }^{1}$ Advanced Centre for Treatment, Research and Education in Cancer, Kharghar, Navi Mumbai, India. ${ }^{2}$ Department of Gastrointestinal Surgery, Gastrointestinal Oncology, and Bariatric Surgery, Medanta Institute of Digestive and Hepatobiliary Sciences, Medanta, The Medicity, Gurgaon, India. ${ }^{3}$ Department of Pathology, Tata Memorial Centre, Ernest Borges Marg, Parel, Mumbai, India. ${ }^{4}$ Department of Gastrointestinal and Hepato-Pancreato-Biliary Surgical Oncology, Tata Memorial Centre, Ernest Borges Marg, Parel, Mumbai, India.

Received: 10 December 2015 Accepted: 23 February 2016

Published online: 03 March 2016

\section{References}

1. Hundal R, Shaffer EA. Gallbladder cancer: epidemiology and outcome. Clin Epidemiol. 2014;6:99-109.

2. Barreto SG, Pawar S, Shah S, Talole S, Goel M, Shrikhande SV. Patterns of failure and determinants of outcomes following radical re-resection for incidental gallbladder cancer. World J Surg. 2014;38(2):484-9.

3. Scanu T, Spaapen RM, Bakker JM, Pratap CB, Wu LE, Hofland I, Broeks A, Shukla VK, Kumar M, Janssen $H$, et al. Salmonella manipulation of host signaling pathways provokes cellular transformation associated with gallbladder carcinoma. Cell Host Microbe. 2015;17(6):763-74.

4. Nagaraja V, Eslick GD. Systematic review with meta-analysis: the relationship between chronic Salmonella typhi carrier status and gall-bladder cancer. Aliment Pharmacol Ther. 2014;39(8):745-50.

5. Dutta U, Garg PK, Kumar R, Tandon RK. Typhoid carriers among patients with gallstones are at increased risk for carcinoma of the gallbladder. Am J Gastroenterol. 2000;95(3):784-7.

6. Caygill CP, Hill MJ, Braddick M, Sharp JC. Cancer mortality in chronic typhoid and paratyphoid carriers. Lancet. 1994;343(8889):83-4.

7. Mager DL. Bacteria and cancer: cause, coincidence or cure? A review. J Transl Med. 2006:4:14.

8. Crump J, Sjölund-Karlsson M, Gordon M, Parry C. Epidemiology, clinical presentation, laboratory diagnosis, antimicrobial resistance, and antimicrobial management of invasive Salmonella infections. Clin Microbiol Rev. 2015;28(4):901-37.

9. Saravanan S, Purushothaman V, Murthy TR, Sukumar K, Srinivasan P, Gowthaman V, Balusamy M, Atterbury R, Kuchipudi SV. Molecular epidemiology of Nontyphoidal Salmonella in poultry and poultry products in India: implications for human health. Indian J Microbiol. 2015;55(3):319-26.

10. Barreto SG, Dutt A, Chaudhary A. A genetic model for gallbladder carcinogenesis and its dissemination. Ann Oncol. 2014;25(6):1086-97.

11. Chandrani P, Kulkarni V, lyer P, Upadhyay P, Chaubal R, Das P, Mulherkar R, Singh R, Dutt A. NGS-based approach to determine the presence of HPV and their sites of integration in human cancer genome. Br J Cancer. 2015;112(12):1958-65.

12. Song $\mathrm{JH}$, Cho H, Park MY, Na DS, Moon HB, Pai CH. Detection of Salmonella typhi in the blood of patients with typhoid fever by polymerase chain reaction. J Clin Microbiol. 1993;31(6):1439-43.

13. Kostic AD, Gevers D, Pedamallu CS, Michaud M, Duke F, Earl AM, Ojesina Al, Jung J, Bass AJ, Tabernero J, et al. Genomic analysis identifies association of Fusobacterium with colorectal carcinoma. Genome Res. 2012;22(2):292-8.

14. Marshall BJ, Warren JR. Unidentified curved bacilli in the stomach of patients with gastritis and peptic ulceration. Lancet. 1984;1(8390):1311-5.

15. Wu XS, Shi LB, Li ML, Ding Q, Weng H, Wu WG, Cao Y, Bao RF, Shu YJ, Ding QC, et al. Evaluation of two inflammation-based prognostic scores in patients with resectable gallbladder carcinoma. Ann Surg Oncol. 2014;21(2):449-57. 